\title{
Influencia de estilos de aprendizaje y roles de equipo en el aprendizaje colaborativo soportado por computadoras
}

\author{
Matías Nicoletti, José María Balmaceda, Silvia Schiaffino y Daniela Godoy \\ ${ }^{1}$ ISISTAN (CONICET - UNCPBA) Campus Universitario, Paraje Arroyo Seco \\ Tandil, B7001BBO, Argentina \\ \{matias.nicoletti, silvia.schiaffino, jose.balmaceda, daniela.godoy\} \\ @isistan.unicen.edu.ar
}

\begin{abstract}
A key aspect when students have to work in groups in a computer supported collaborative learning context is how the groups are formed. In this article, we analyze how two types of students' characteristics might influence on the success or the failure of the collaborative learning process when students have to work in groups. Particularly, we study how students' learning styles and students' team roles might affect the performance of the team. By applying Artificial Intelligence techniques we have discovered interesting relationships among these students' characteristics and team performance. We consider that these findings can shed some light to the area of group formation in the area of computer supported collaborative learning.
\end{abstract}

\section{Introducción}

El aumento de la accesibilidad de Internet en los últimos años ha favorecido el uso de herramientas de educación a distancia. En este contexto, uno de los tipos de aplicaciones más utilizadas son las herramientas de aprendizaje colaborativo (CSCL), las cuales tienen como principal objetivo proveer un ambiente de trabajo virtual en la cual personas que se encuentran distantes físicamente puedan compartir y construir nuevo conocimiento mediante el uso de la computadora [Bannon and Schmidt 1991]. Sin embargo, la forma en que se agrupan los alumnos puede afectar el desempeño grupal. Un concepto clave para conseguir resultados positivos en entornos grupales es la noción de cooperación, la cual se refiere a la forma en la que los individuos trabajan interdependientemente y contribuyen esfuerzos para conseguir beneficios mutuos a través de la participación activa y la comunicación entre éstos. El trabajo colaborativo, además, implica compartir habilidades, experiencias y responsabilidades individuales en propósito de un objetivo común.

Existen varios factores que pueden influenciar el desempeño de un grupo de aprendizaje. Por ejemplo, la manera en la que los estudiantes aprenden: viendo y escuchando; reflexionando y actuando; razonando lógica e intuitivamente; memorizando y visualizando; haciendo analogías y construyendo modelos matemáticos [Felder and Silverman 1988]. Un modelo de estilos de aprendizaje clasifica a los estudiantes de acuerdo a cómo encajan en un número de categorías que corresponden a la forma en que reciben y procesan información.

Por otro lado, las teorías de roles de equipo sugieren que los miembros de un equipo siguen patrones de comportamiento característicos denominados roles de equipo. Cada rol de equipo describe una forma de interactuar con los otros integrantes y la manera 
en la cual aporta al progreso del equipo [Belbin 1993]. Dentro de las distintas teorías de roles de equipo existentes en la literatura, la propuesta por Belbin está compuesta de nueve roles de equipo y asegura que el desempeño grupal será mejor cuando más balanceados se encuentros dichos roles dentro del equipo. En consecuencia, la distribución de los roles de equipo de los integrantes es un característica que también podría incidir en el desempeño de grupos de aprendizaje.

En este contexto, nuestro objetivo es analizar si algunas características específicas de los estudiantes influyen en el desempeño del grupo. Nuestra suposición es que el estilo de aprendizaje y la distribución de los roles de equipos dentro del grupo puede afectar el trabajo y el aprendizaje grupal. Para modelar los estilos de aprendizaje utilizamos el modelo formulado por Felder y Silverman debido a que ha sido evaluado exitosamente en diferentes dominios [Felder and Brent 2005]. De forma similar, para modelar los roles de equipo utilizamos la teoría propuesta por Meredith Belbin, reconocida como una de las teorías de roles de equipos más difundida y validada [Aritzeta et al. 2007].

Para verificar nuestra hipótesis utilizamos reglas de asociación con el fin de descubrir relaciones de influencia entre las características individuales y el resultado del trabajo en grupo. Se efectuó un experimento en un entorno de aprendizaje colaborativo en un ambiente académico en el cual los alumnos realizaron un ejercicio de aprendizaje y puesta en práctica de nuevos conceptos. Los resultados obtenidos indican que efectivamente estos aspectos tienen un efecto sobre el resultado del trabajo grupal. Además, proponemos algunas ideas para que un docente o una herramienta automática (de CSCL) forme grupos de trabajo o de estudio que se desempeñen de manera satisfactoria.

El resto del artículo se organiza de la siguiente manera. En la Sección 2 se describen los conceptos principales sobre estilos de aprendizaje, sobre roles de equipo y reglas de asociación. En la Sección 3 se detallan algunos trabajos relacionados. En la Sección 4 se detallan en primer lugar, las técnicas utilizadas para descubrir relaciones entre las características de los alumnos y el desempeño en el trabajo colaborativo soportado por computadoras, y por último, los patrones obtenidos. Finalmente, en la Sección 5 se describen las conclusiones a las que hemos arribado.

\section{Marco Teórico}

\subsection{Estilos de Aprendizaje}

Los estilos de aprendizaje son características cognitivas, y comportamientos psicológicos que sirven como indicadores de cómo los estudiantes perciben, interactúan y responden a los distintos ambientes de aprendizaje. Uno de los modelos de estilo de aprendizaje más reconocido es el propuesto por Felder-Silverman [Felder and Silverman 1988], el cual fue diseñado para capturar las diferentes características de los distintos estudiantes de ingeniería y proveer una sólida base para que los profesores puedan armar sus clases según las preferencias de sus alumnos. Según Felder los estilos de aprendizaje son una combinación de 4 dimensiones: comprensión, percepción, entrada y procesamiento. Cada una de estas dimensiones puede tomar uno de dos valores posibles por lo que se tienen 16 estilos de aprendizaje dentro del modelo de Felder. La comprensión puede ser global o secuencial, la percepción puede ser activa o sensitiva, la entrada verbal o visual y el procesamiento puede ser activo o reflexivo. 
De este modo, de acuerdo a los valores obtenidos por los alumnos sobre cada una de estas dimensiones, afecta la forma en la cual prefiere aprender, o tiene tendencia a hacerlo. Por ejemplo, según la dimensión de procesamiento, los alumnos sensitivos tienden a aprender más rápido a través de hechos concretos, resolviendo un gran número de problemas; son estudiantes pacientes, pueden memorizar hechos concretos, son más prácticos y cuidadosos. Por otro lado, los alumnos intuitivos prefieren descubrir y relacionar los nuevos conceptos con conocimientos previos, les gusta innovar, se inclinan hacia toda la información abstracta y las demostraciones teóricas. En referencia a la dimensión de procesamiento, los estudiantes activos no aprenden mucho en situaciones donde ellos deben ser pasivos, como en las lecturas de textos. Por el contrario, estas situaciones son muy favorables para los alumnos reflexivos que necesitan tener la oportunidad de pensar sobre la nueva información.

El modelo de Felder-Silverman ha sido utilizado exitosamente por profesores universitarios y estudiantes de ingenierías y ciencias [Felder and Brent 2005]. Otros ejemplos de modelos de estilos de aprendizaje que se pueden encontrar en la literatura son [Dunn 1990, Honey and Mumford 1992]. En particular, Felder y su equipo desarrollaron una herramienta denominada Indice de Estilos de Aprendizaje (ILS) [Felder and Brent 2005] que permite predecir las preferencia de los alumnos respecto de las 4 dimensiones de su modelo. El índice de estilos de aprendizaje es un cuestionario de 44 preguntas. La primera versión de este cuestionario fue realizada en el año 1991 y después de varias pruebas, en 1994, se obtuvo un nuevo cuestionario que es el que se encuentra en vigencia actualmente. A partir de 1997 este cuestionario ha sido publicado públicamente en la Web ${ }^{1}$.

\subsection{Roles de Equipo}

Belbin [Belbin 1993] fue el primer investigador en plantear una de las teorías de roles de equipo más difundidas en el área de formación de grupos. Con la aparición del concepto de roles de equipo, se manifiesta una importante división en el concepto entre roles funcionales y roles de equipo. Los roles funcionales abarcan las acciones físicas, actividades intelectuales, y objetivos específicos relacionados al trabajo de una persona. Por otro lado, los roles de equipo describen la naturaleza de las contribuciones que una persona realiza en beneficio del grupo. Estas contribuciones generalmente no son evidentes y no están asociadas a ningún requerimiento de trabajo. Los roles de equipo son adoptados naturalmente por las personas debido a su personalidad y comportamientos aprendidos, más que por capacidades técnicas y conocimientos específicos de una tarea.Además del modelo de Belbin, existen otro modelos tales como Mumma [Mumma 1992], MTR-i² Insights $^{3}$, Thomas International ${ }^{4}$, entre otras.

Meredith Belbin [Belbin 1993] identificó nueve roles de equipo y afirmó que la efectividad de los equipos de trabajo está directamente relacionada con la existencia de diversos Roles en los equipos de trabajo. Posteriormente, continuando con sus investigaciones, Belbin afirmó que un equipo de trabajo puede ser coordinado si existe un equilibrio adecuado de roles [Belbin 2001]. Dicho equilibrio aparece cuando en un grupo se

\footnotetext{
${ }^{1}$ http://www.engr.ncsu.edu/learningstyles/ilsweb.html

${ }^{2} \mathrm{http}: / /$ www.profiles-r-us.com/mtri/

${ }^{3}$ http://www.insights.com/index.aspx

${ }^{4}$ http://www.thomasinternational.net/
} 
manifiesta la mayor cantidad de roles posibles, y los mismos no se repiten entre los integrantes del grupo. El modelo de Belbin esta compuesto por los siguientes 9 roles: Cerebro, Investigador de recursos, Coordinador, Impulsor, Monitor-Evaluador, Cohesionador, Implementador, Finalizador y Especialista.

La mayoría de las teorías de roles de equipo han desarrollado diferentes herramientas para la detección del rol o los roles adecuados para ser asignados a una determinada persona. Estas herramientas también pueden convertirse en importantes instrumentos de autoayuda para descubrir las habilidades actuales y potenciales de la persona. Particularmente, Belbin desarrolló el Inventario de Belbin de Auto-Percepción de Rol de Equipo (BTRSPI-9R, del inglés Belbin Team-Role Self-Perception Inventory), un cuestionario compuesto de 7 secciones, cada una formada por 10 ítems que muestran diferentes situaciones de trabajo en equipo.

\subsection{Reglas de asociación}

Una regla de asociación es una regla que implica una relación de asociación entre un conjunto de objetos (o ítems) en una base de datos, tal como "ocurren juntos" o "uno implica el otro". De manera más formal, el problema de descubrir reglas de asociación generalmente se expresa como sigue [Agrawal and Srikant 1994]: Sea $I=i_{1}, \ldots, i_{n}$ un conjunto de elementos, y sea $D$ un conjunto de instancias. Cada instancia consiste de un subconjunto de elementos de $I$. Una regla de asociación es una implicación de la forma $X \rightarrow Y$, donde $X \subseteq I, Y \subseteq I$, y $X \cap Y=\phi$. Xes el antecedente de la regla e $Y$ es su consecuente. El soporte de una regla $X \rightarrow Y$ es la probabilidad de que los conjuntos $X$ e $Y$ ocurran juntos en la misma transacción. La regla tiene soporte $s$ en $D$ si $s \%$ de las instancias de $D$ contienen $X \cup Y$. La regla $X \rightarrow Y$ tiene una confianza $c$ en $D$ si $c \%$ de las instancias en $D$ que contienen a $X$ también contienen a $Y$. La confianza de una regla $X \rightarrow Y$ se define como la probabilidad de ocurrencia de $X$ e $Y$ juntos en todas las transacciones en las que ya aparece $X$.

Dada una base de datos $D$, el problema de descubrir reglas de asociación es encontrar todas las reglas que satisfagan los criterios de soporte y confianza mínimos establecidos por el usuario. Se han realizado numerosos trabajos en el área de reglas de asociación, y como resultado, existen diversos algoritmos para el descubrimiento de reglas. El más popular es el algoritmo Apriori [Agrawal and Srikant 1994], que es el que utilizamos para descubrir patrones en este trabajo.

\section{Trabajos Relacionados}

Algunos investigadores han analizado la forma en que los estilos de aprendizaje impactan la forma de agrupar alumnos en trabajo colaborativo. Uno de los modelos de estilos de aprendizaje más utilizados es el propuesto por Felder-Silverman [Felder and Silverman 1988]. Por ejemplo, este modelo es aplicado en [Alfonseca et al. 2006, Martin and Paredes 2004, Monteserin et al. 2010]. El primer trabajo explora los efectos que puede producir el hecho de combinar estudiantes con diferentes estilos de aprendizaje sobre los resultados del trabajo en grupo. Por el otro lado, en [Martin and Paredes 2004] se propone utilizar el modelo de Felder-Silverman para formar grupos heterogéneos y adaptar automáticamente las actividades propuestas en un sistema Web. En [Monteserin et al. 2010] se analiza la relación entre estilos de aprendi- 
zaje y habilidades de negociación en el resultado del trabajo en grupo en contextos de e-learning.

Otro aspecto evaluado en la formación de grupos es la personalidad de los integrantes. Por ejemplo, en [Speck 2003] se presenta un modelo para la formación de grupos basándose en diferentes tests de personalidad. El modelo se validó experimentalmente utilizando grupos de alumnos con diferentes personalidades. Con respecto a los roles de equipo, pocos trabajos han a considerado ésta característica para el aprendizaje colaborativo. Algunos trabajos recientes en esta dirección son [Costaguta et al. 2012, Fares et al. 2012], donde se proponen enfoques para mantener el balance de roles de equipo en grupos en contextos de CSCL.

\section{Resultados Experimentales}

\subsection{Descripción del conjunto de datos}

Para realizar el experimento se recolectó un conjunto de datos correspondiente al trabajo grupal realizado por alumnos de la carrera Ingeniería de Sistemas de la UNCPBA (Universidad Nacional del Centro de la Pcia. De Bs. As., Argentina) durante una materia de 3er año. Participaron 93 alumnos, de los cuales 13 eran mujeres (14\%) y 80 eran varones (86\%). Los alumnos fueron divididos en 31 grupos de 3 integrantes cada uno, y debían resolver un trabajo práctico requerido para la aprobación de la materia. Los datos fueron obtenidos mediante el monitoreo de las acciones de los usuarios al utilizar un software de CSCW (o Groupware). A continuación se describen los diferentes atributos del conjunto de datos utilizado, donde cada fila representa un grupo.

- Nivel de interacción grupal: Grado de participación general que tuvo el grupo al que el alumno pertenece. ${ }^{5}$

- Nivel de coordinación grupal: Grado de organización y coordinación general del grupo para la realización de tareas durante la resolución del ejercicio. ${ }^{5}$

- Nivel de conflictos grupal: Representa el grado de conflictos y discusiones entre los integrantes del grupo. ${ }^{5}$

- Tipo de Grupo Percepción: Indica si el grupo era homogéneo (intuitivo, neutral o sensitivo) o heterogéneo de acuerdo al estilo de aprendizaje de Percepción.

- Tipo de Grupo Procesamiento: Indica si el grupo era homogéneo (activo, neutral o reflexivo) o heterogéneo de acuerdo al estilo de aprendizaje de Procesamiento.

- Tipo de Grupo Rol: Indica si el grupo era homogéneo o heterogéneo de acuerdo a los roles de equipo involucrados.

- Calificación: Calificación obtenida por el grupo. Los valores que puede tomar este atributo son Regular, Bien o Muy Bien.

Los niveles de interacción, coordinación y conflictos para un grupo dado (medidos de forma grupal) se calculan en base a la cantidad de acciones efectuadas por los miembros de dicho grupo y en relación con la cantidad de acciones de otros grupos. Para determinar el tipo de una acción (interacción, coordinación o conflicto) se utilizaron sentencias de apertura . Por otro lado, para detectar los estilos de aprendizaje y los roles de equipo, se le solicitó a los alumnos que respondieran los cuestionarios ILS de Felder y BTRSPI-9R de Belbin, respectivamente.

\footnotetext{
${ }^{5}$ Se mide en una escala del 1 al 5, donde 5 es el máximo.
} 
Se efectuó un pre-procesamiento de algunas variables con el objetivo de simplificar/mejorar el proceso de extracción de reglas. Las variables Nivel de Coordinación y Nivel de Interacción se discretizaron de la siguiente forma: [1,3] como Regular, 4 como Bien y 5 como Muy Bien. De forma similar, la variable Nivel de Conflictos se discretizó de la siguiente forma: [3,5] como Regular, 2 como Bien y 1 como Muy Bien.

En cuanto a la distribución de la población respecto de las características consideradas, en la Figura 1 se muestra la distribución correspondiente a los roles de equipo de los alumnos. Similarmente, en la Figura 2 se muestra la distribución correspondiente a los estilos de aprendizaje, tanto para las dimensiones de percepción y de procesamiento.

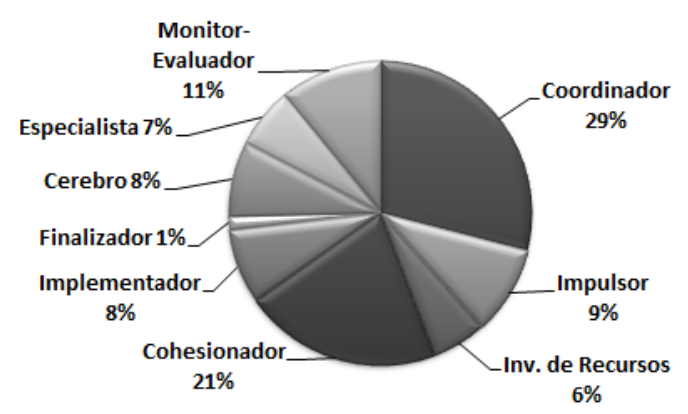

Figura 1. Distribución de roles de equipo.
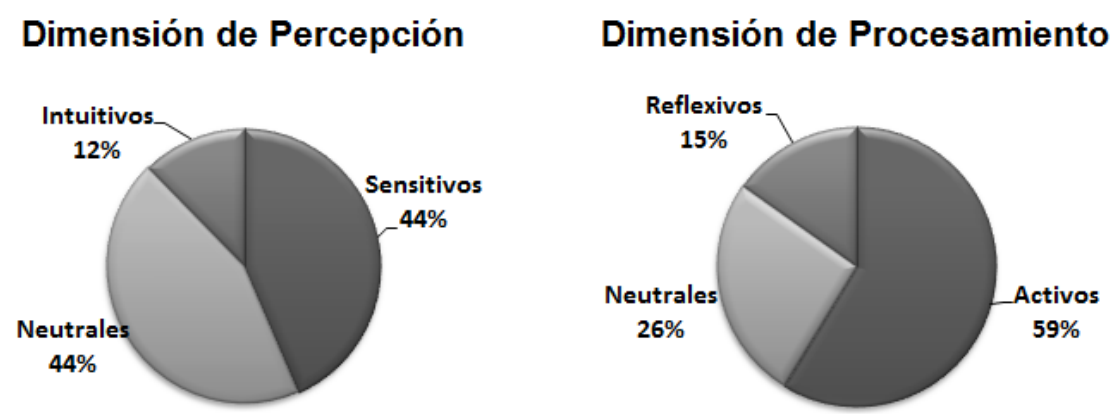

Figura 2. Distribución de estilos de aprendizaje.

\subsection{Descripción del Experimento}

El objetivo de este experimento es encontrar relaciones significativas de dependencia entre las configuraciones de grupos (en cuanto a estilos de aprendizaje y roles de equipo) y el desempeño general que manifestaron. Para lograr dicho objetivo, se plantearon dos preguntas de investigación: (1) ¿Qué combinación de estilos de aprendizaje dio mejores resultados, considerando la calificación y el desempeño grupal? y (2) ¿Cuál sería una configuración recomendable para formar un equipo de trabajo, si se conociesen tanto los estilos de aprendizaje como los roles de los candidatos a ser integrantes?. Posteriormente, se ejecutó el algoritmo Apriori sobre el conjunto de datos y se analizaron las relaciones resultantes en búsqueda de evidencia que nos permita concluir sobre las preguntas planteadas. La diferencia en el análisis para cada pregunta se encuentra en la inclusión de la variable Tipo de Grupo Rol en el algoritmo Apriori (utilizada en la pregunta 2).

Debido a que en este trabajo nos interesan aquellas reglas de asociación que posean en el consecuente el atributo Calificación y también aquellas que posean los atributos Nivel de Interacción y/o Nivel de Coordinación y/o Nivel de Conflictos, se realizó 
un pos-procesamiento de las reglas. En primer lugar, se aplicó un filtro de reglas de asociación mediante plantillas [Klemettinen et al. 1994]. Una plantilla indica qué tipos de atributos se espera que aparezcan en el antecedente de la reglas y/o qué tipos de atributos se espera que aparezcan en el consecuente de las reglas. Una plantilla tiene la forma: $A_{1}, \ldots, A_{k} \rightarrow A_{k+1}$, donde cada $A_{i}$ es un atributo. Una regla $B_{1}, \ldots, B_{h} \rightarrow B_{h+1}, \ldots, B_{m}$ se corresponde con una plantilla, si la regla puede considerarse como una instancia de la plantilla. Por otro lado, debido a la posible existencia de reglas redundantes, se realizó un filtrado de reglas siguiendo el método propuesto por Liu et al. [Liu et al. 1999]: dada las reglas $R_{1}: X \rightarrow Y$ y $R_{2}: X, Z \rightarrow Y$ con valores de confianza similares, $R_{2}$ puede considerarse redundante con respecto a $R_{1}$ pues no aporta nueva información. De manera similar, dadas las reglas $R_{1}: X \rightarrow Y$ y $R_{2}: X \rightarrow Y, Z$, se considera que la regla $R_{1}$ es redundante respecto a $R_{2}$ y debe eliminarse.

\subsection{Resultados}

Con respecto a la Pregunta 1, no se observaron relaciones significativas entre la combinación de estilos de aprendizaje y la Calificación obtenida. Las reglas obtenidas poseen confianzas muy bajas, menores al $50 \%$. En cuanto al Nivel de Interacción, se observa que tuvieron muy buen desempeño aquellos grupos Homogéneos - Sensitivos (Percepción), Homogéneos - Neutrales (Procesamiento), y la combinación de estilos Homogéneos Neutrales (Percepción) y Heterogéneos (Procesamiento). En cuanto al Nivel de Coordinación, se observa que tuvieron muy buen desempeño aquellos grupos Homogéneos - Neutrales (Procesamiento), Heterogéneos (Procesamiento) y Homogéneos - Sensitivos (Percepción). En cuanto al Nivel de Conflictos, se observa que tuvieron baja tasa de conflictos aquellos grupos Heterogéneos (Procesamiento), Homogéneos - Neutrales (Percepción), Homogéneos - Neutrales (Procesamiento) y Homogéneos - Sensitivos (Percepción). Algunos ejemplos de las reglas obtenidas se muestran en la Figura 3.

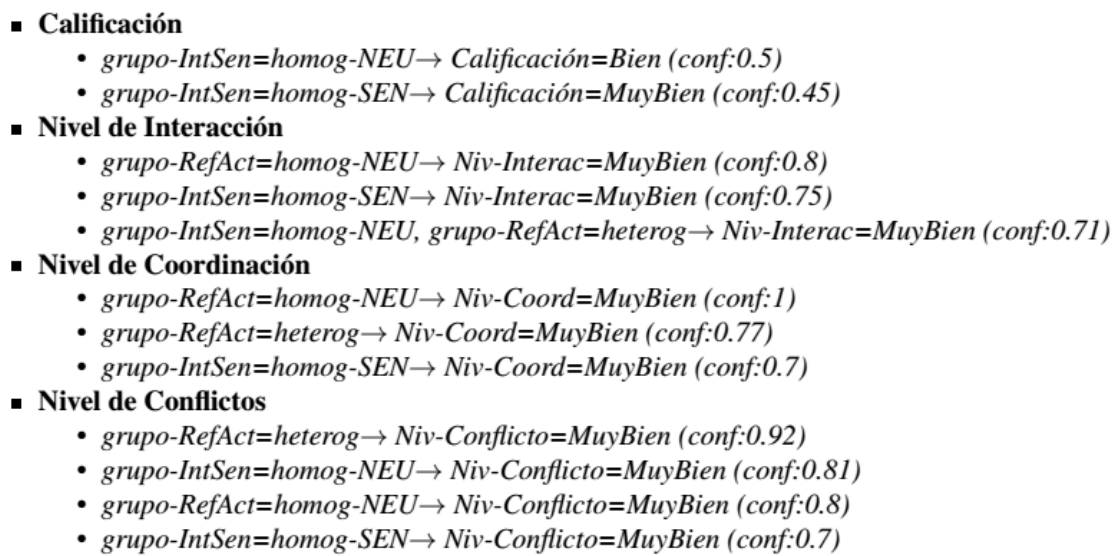

Figura 3. Reglas de asociación para la Pregunta 1.

Las reglas descubiertas pueden interpretarse de la siguiente forma. Por ejemplo, la primer regla (Calificación) indica que aquellos grupos homogéneos neutrales respecto a la dimensión percepción de los estilos de aprendizaje, obtuvieron buenas calificaciones con una confianza de $50 \%$. Por otro lado, la quinta regla (Nivel de Interacción) indica que aquellos grupos homogéneos neutrales en la dimensión percepción y heterogéneos en la 
dimensión procesamiento, se desempeñaron con muy buenos niveles de interacción con una confianza del $71 \%$.

Posteriormente se procedió a analizar las reglas de asociación para la Pregunta 2 (referida a configuraciones de grupos recomendables en base a los estilos y roles). En cuanto a la Calificación, se observó que obtuvieron muy buen desempeño aquellos grupos Homogéneos-Coordinador (en cuanto al rol de sus integrantes). En cuanto al Nivel de Interacción, se observa muy buen desempeño de aquellos grupos Heterogéneos y Homogéneos-Coordinador. En cuanto al Nivel de Coordinación, se observa muy desempeño de aquellos grupos Heterogéneos. Con respecto al Nivel de Conflictos, se observa que tuvieron baja tasa de conflictos aquellos grupos Heterogéneos. En la Figura 4 se muestran ejemplos de reglas reflejando lo descripto anteriormente.

- Calificación

- grupo-IntSen=homog-SEN, grupo-Rol=homog-COOR $\rightarrow$ Calificación=MuyBien (conf:0.83)

- grupo-Rol=homog-COOR $\rightarrow$ Calificación=MuyBien (conf:0.67)

- Nivel de Interacción

- grupo-IntSen=homog-SEN, grupo-RefAct=homog-ACT, grupo-Rol=heterog $\rightarrow$ Niv-Interac $=$ MuyBien (conf:0.88)

- grupo-Rol=homog-COOR $\rightarrow$ Niv-Interac $=$ MuyBien ( conf:0.78)

- Nivel de Coordinación

- grupo-RefAct=heterog, grupo-Rol=heterog $\rightarrow$ Niv-Coord=MuyBien ( conf: $(0.84)$

- grupo-Rol=heterog $\rightarrow$ Niv-Coord=MuyBien (conf:0.73)

- Nivel de Conflictos

- grupo-IntSen=homog-NEU, grupo-RefAct=heterog, grupo-Rol=heterog $\rightarrow$ Niv-Conflicto $=$ MuyBien (conf: 1$)$

- grupo-Rol=heterog $\rightarrow$ Niv-Conflicto=MuyBien (conf:0.73)

Figura 4. Reglas de asociación para la Pregunta 2.

En ambos análisis, la confianza mínima fue del $70 \%$ y el soporte mínimo fue del $10 \%$. Los resultados previos pueden resumirse en la Tabla 1, donde se marcan los tipos de grupos que mostraron los mejores desempeños según cada variable observada.

\begin{tabular}{|l|c|c|c|c|}
\hline \multirow{2}{*}{ Tipo de Grupo } & \multicolumn{4}{|c|}{ Aspectos de desempeño } \\
\cline { 2 - 5 } & $\begin{array}{c}\text { Nivel de } \\
\text { Interacción }\end{array}$ & $\begin{array}{c}\text { Nivel de } \\
\text { Coordinación }\end{array}$ & $\begin{array}{c}\text { Nivel de } \\
\text { Conflictos }\end{array}$ & Calificación \\
\hline Hom. Sensitivos & $\mathrm{X}$ & $\mathrm{X}$ & $\mathrm{X}$ & \\
\hline Hom. Neutrales Per. & $\mathrm{X}$ & & $\mathrm{X}$ & \\
\hline Hom. Intruitivos & & & & \\
\hline Heterogéneo Per. & & & & \\
\hline Hom. Activos & & & $\mathrm{X}$ & \\
\hline Hom. Neutrales Pro. & $\mathrm{X}$ & $\mathrm{X}$ & $\mathrm{X}$ & \\
\hline Hom. Reflexivo & & & $\mathrm{X}$ & $\mathrm{X}$ \\
\hline Heterogéneo Pro. & $\mathrm{X}$ & $\mathrm{X}$ & $\mathrm{X}$ & \\
\hline Hom. Coordinador & $\mathrm{X}$ & & $\mathrm{X}$ & $\mathrm{X}$ \\
\hline Heterogéneo Rol & $\mathrm{X}$ & $\mathrm{X}$ & & \\
\hline
\end{tabular}

Tabla 1. Resumen de relaciones encontradas.

\subsection{Discusión}

Los resultados resumidos en la Tabla 1 pueden servir como guía a docentes para armar grupos de trabajo en contextos de aprendizaje colaborativo soportado por computadoras. Conociendo los estilos de aprendizaje de los alumnos y los roles de equipo que prefieren desempeñar, pueden armarse grupos que respondan a los patrones mencionados previamente, dado que poseen altas probabilidades de desempeñarse de manera satisfactoria. 
Algunos de los patrones descubiertos coinciden con lo esperado de acuerdo a las teorías de roles de equipo. Por ejemplo, las reglas descubiertas indican que se han desempeñado de manera muy satisfactoria aquellos grupos heterogéneos respecto a los roles de equipo que manifiestan sus integrantes. Esto coincide con la propuesta de Belbin. En consecuencia, nuestro trabajo evidencia que las teorías de roles de equipo aplican también en contextos educativos. Adicionalmente, se destaca que formar equipos con mayoría de roles de Coordinador podría tener resultados satisfactorios.

Debe considerarse que algunos patrones descubiertos pueden haberse debido a características particulares del conjunto de datos utilizados. Si se observan las distribuciones de la población, hay mayoría de alumnos Activos (Procesamiento), Sensitivos y Neutrales (Percepción), y gran cantidad de alumnos con los roles de de Coordinador y Cohesionador. Estas características pueden haber ocasionado que se descubran patrones donde predominen estos atributos. Sin embargo, dichas consideraciones solo afectarían a una fracción de los resultados resumidos en la Tabla 1. Por ejemplo, el hecho de haber detectado un excelente desempeño de los grupos Homogéneos - Sensitivos puede deberse a la mayoría de alumnos con dicho estilo, pero haber detectado que los grupos Homogéneos - Neutrales (Procesamiento) tuvieron un desempeño similar es un patrón interesante, considerando que dicho estilo es minoritario.

\section{Conclusiones}

En este artículo hemos presentado un análisis de la influencia de los estilos de aprendizaje y roles de equipo de los alumnos, sobre el resultado del trabajo grupal realizado en contextos de CSCW. Los resultados obtenidos utilizando reglas de asociación han permitido determinar relaciones entre dichas características y el desempeño de los grupos de trabajo. Los hallazgos de nuestro estudio pueden ser utilizados tanto por docentes como por herramientas automáticas para generar grupos de trabajo que garanticen un buen desempeño. El desempeño grupal se ha medido teniendo en cuenta factores como la calificación, el nivel de coordinación, el nivel de interacción y el nivel de conflictos durante la resolución de un trabajo práctico en un contexto educativo. Por este motivo, creemos que este trabajo efectúa una contribución importante al área de educación.

Como trabajo futuro, se utilizarán otras técnicas de Inteligencia Artificial y/o análisis estadísticos para analizar el problema planteado. Además, se recolectarán nuevos conjuntos de datos que permitan replicar el estudio y corroborar los resultados de esta experiencia. También se planea incorporar otros factores al análisis que puedan afectar el desempeño de los estudiantes, tales como como la personalidad y factores emocionales.

\section{Agradecimientos}

Este trabajo fue parcialmente financiado por ANPCyT (Argentina) a través del proyecto PICT $2011 \mathrm{~N}^{\circ} 0366$.

\section{Referencias}

Agrawal, R. and Srikant, R. (1994). Fast algorithms for mining association rules. In Proc. 20th Int. Conf. Very Large Data Bases, VLDB, volume 1215, pages 487-499.

Alfonseca, E., Carro, R., Martin, E., Ortigosa, A., and Paredes, P. (2006). The impact of learning styles on student grouping for collaborative learning: a case study. User Modeling and User-Adapted Interaction, 16(3-4):377-401. 
Aritzeta, A., Swailes, S., and Senior, B. (2007). Belbin's team role model: Development, validity and applications for team building. J. of Management Studies, 44(1):96-118.

Bannon, L. J. and Schmidt, K. (1991). Cscw: Four characters in search of a context. In Studies in Computer Supported Cooperative Work, volume 8 of Human Factors in Information Technology, pages 3-17. North-Holland, Amsterdam, Netherlands.

Belbin, R. M. (1993). Team roles at work. Oxford: Butterwoth-Heinemann.

Belbin, R. M. (2001). Managing without power. Oxford: Butterworth-Heinemann.

Costaguta, R., Schiaffino, S., and Fares, R. (2012). New Horizons in Creative Open Software, Multimedia, Human Factors and Soft. Engineering, chapter Fostering team-role balance in Computer Supported Collaborative Learning, pages 82-90. Blue Herons.

Dunn, R. (1990). Understanding the dunn and dunn learning styles model and the need for individual diagnosis and prescription. Journal of Reading, Writing, and Learning Disabilities, 6:223-247.

Fares, R., Costaguta, R., Schiaffino, S., and Missio, D. (2012). Grupos de aprendizaje colaborativo asistidos por agentes para promover el equilibrio de roles. Workshop de Investigadores en Ciencias de la Computacion, WICC 2012, pages 83-87.

Felder, R. M. and Brent, R. (2005). Understanding student differences. Journal of Engineering Education, 94:57-72.

Felder, R. M. and Silverman, L. K. (1988). Learning and teaching styles in engineering education. Engineering education, 78(7):674-681.

Honey, P. and Mumford, A. (1992). The Manual of Learning Styles. 3rd Ed. Maidenhead.

Klemettinen, M., Mannila, H., Ronkainen, P., Toivonen, H., and Verkamo, A. I. (1994). Finding interesting rules from large sets of discovered association rules. In Proceedings of the third international conference on Information and knowledge management, CIKM '94, pages 401-407, New York, NY, USA. ACM.

Liu, B., Hsu, W., and Ma, Y. (1999). Pruning and summarizing the discovered associations. In Proceedings of the 5th ACM SIGKDD International Conference on Knowledge Discovery and Data Mining, pages 125-134, New York, USA. ACM.

Martin, E. and Paredes, P. (2004). P.: Using learning styles for dynamic group formation in adaptive collaborative hypermedia systems. In Proceedings of the First International Workshop on Adaptive Hypermedia and Collaborative Web-based Systems.

Monteserin, A., Schiaffino, S., Garcia, P., and y Amandi, A. (2010). Análisis de la formación de grupos en aprendizaje colaborativo soportado por computadoras. Anais do XXI Simposio Brasileiro de Informatica na Educação, SBIE 2010.

Mumma, F. S. (1992). Team-work and Team-roles: What Makes Your Team Tick? Organization Design and Development.

Speck, B. W. (2003). Fostering collaboration among students in problem-based learning. New Directions for Teaching and Learning, 2003(95):59-65. 